\title{
1 Antiviral RNAi response against the insect-specific Agua Salud
}

\section{2 alphavirus}

3 Mine Altinli $^{1 \S}$, Mayke Leggewie ${ }^{1 \S}$, Marlis Badusche ${ }^{1}$, Rashwita Gyanwali $^{1}$, Christina Scherer $^{1}$, Jonny Schulze $^{1}$,

4 Vattipally B. Sreenu ${ }^{2}$, Marvin Fegebank ${ }^{1}$, Bernhard Zibrat ${ }^{1}$, Janina Fuss ${ }^{3}$, Sandra Junglen ${ }^{4}$, Esther Schnettler $^{1,5 *}$

$6 \quad{ }^{1}$ Bernhard Nocht Institute for Tropical Medicine, Hamburg, Germany, and German Centre for Infection

7 Research (DZIF), partner site Hamburg-Luebeck-Borstel-Riems, Germany

$8 \quad{ }^{2}$ MRC-University of Glasgow-Centre for virus Research, Glasgow, UK

$9 \quad{ }^{3}$ Institute of Clinical Molecular Biology (IKMB), Kiel University, Kiel, Germany

$10{ }^{4}$ Institute of Virology, Charité - Universitätsmedizin Berlin, corporate member of Free University Berlin,

11 Humboldt-University Berlin, and Berlin Institute of Health, Berlin, Germany

$12{ }^{5}$ University Hamburg, Faculty of Mathematics, Informatics and Natural Sciences, 20148 Hamburg, Germany

$15 \S$ contributed equally to the work

16 * Correspondence: schnettler@bnitm.de

\section{Abstract:}

21 Arboviruses transmitted by mosquitoes are responsible for the death of millions of people each year.

22 In addition to arboviruses, many insect-specific viruses (ISVs) have been discovered in mosquitoes in 23 the last decade. ISVs, in contrast to arboviruses transmitted by mosquitoes to vertebrates, cannot 


\section{Introduction} alphaviruses.

replicate in vertebrate cells even when they are evolutionarily closely related to arboviruses. The alphavirus genus includes many arboviruses, although only a few ISVs have been discovered from this genus so far. Here, we investigate the interactions of a recently isolated insect-specific alphavirus, Agua-Salud alphavirus (ASALV), with its mosquito host.

RNAi is one of the essential antiviral responses against arboviruses, although there is little knowledge on the interactions of RNAi with ISVs. Through knock-down of transcripts of the different key RNAi pathway (siRNA, miRNA and piRNA) proteins, we show the antiviral role of Ago2 (siRNA), Ago1 (miRNA), and Piwi4 proteins against ASALV in Aedes aegypti derived cells. ASALV replication increased in Dicer2 and Ago2 knock-out cells, confirming the antiviral role of the siRNA pathway. In infected cells, mainly ASALV-specific siRNAs are produced while piRNAs, with the characteristic nucleotide bias resulting from ping-pong amplification, are only produced in Dicer2 knock-out cells. Taken together, ASALV interactions with the mosquito RNAi response differs from arthropod-borne alphaviruses in some aspects, although they also share some commonalities. Further research is needed to understand whether the identified differences can be generalised to other insect-specific

Mosquitoes are efficient vectors for many medically important arthropod-borne viruses (arboviruses) from several RNA virus families such as Flaviviridae, Togaviridae, Bunyavirales, Reoviridae, and Rhabdoviridae (Weaver \& Reisen, 2010). Arboviruses have a complex life cycle consisting of replication in both vertebrate and invertebrate hosts. In the last decade, many viruses that are restricted to invertebrate hosts (i.e. that cannot replicate in vertebrate hosts) have also been discovered (Atoni et al., 2019). These viruses, generally termed insect-specific viruses (ISVs), have been discovered from all major arbovirus families. They are considered promising for many applications, from vaccine development to arbovirus transmission control tools (Agboli et al., 2019). Nevertheless, our knowledge 
of many important aspects of ISV biology is limited, such as their interactions with the vector species they infect (Altinli et al., 2021).

Arboviruses establish asymptomatic persistent infections in mosquito vectors which are attributed to the efficiency of the mosquito innate immune system. As a part of the mosquito innate immune system, RNA interference (RNAi) pathways play a major role in regulating arbovirus infections (Donald et al., 2012; Leggewie \& Schnettler, 2018). There are three RNAi pathways in mosquitoes: micro (mi)RNA, small interfering (si)RNA and P-element induced wimpy testis (PIWI)-interacting (pi)RNA pathways (Donald et al., 2012; Leggewie \& Schnettler, 2018). The siRNA pathway is triggered by double-stranded (ds)RNA and categorised as exogenous or endogenous depending on the origin of the dsRNA. Among these, the exogenous (exo-)siRNA pathway is considered the primary antiviral defence mechanism for mosquitoes and other insects (Bronkhorst \& Van Rij, 2014; Leggewie \& Schnettler, 2018). The exo-siRNA pathway can be induced by dsRNA derived either from viral replication or RNA secondary structures, which are cut by Dicer2 (Dcr2) into virus-derived siRNAs (vsiRNA), that are 21 nucleotides in length (Donald et al., 2012; Leggewie \& Schnettler, 2018). These vsiRNAs are then incorporated into the RNA-induced silencing complex (RISC), specifically to the Argonaute2 (Ago2) protein, and guide the complex to target complementary viral RNA for subsequent cleavage; resulting in the inhibition of virus replication. vsiRNAs specific to arboviruses are produced during infection by all major arboviruses, proving an interaction with the exo-siRNA pathway (Liu et al., 2019). Furthermore, knock-down or knock-out of key players involved in the exo-siRNA pathway, Dcr2 and Ago2 proteins, led to an increase in replication of all tested arboviruses, supporting the antiviral role of these proteins and the exo-siRNA pathway against arboviruses in mosquitoes (Liu et al., 2019; Scherer et al., 2021).

The miRNA pathway is known to regulate gene expressions of endogenous transcripts in various organisms, including mosquitoes. The miRNA pathway starts by cleaving primary miRNAs into precursor (pre-)miRNA molecules in the nucleus. After exportation into the cytoplasm, pre-miRNA is cut to miRNA/miRNA* duplexes of 21-22nt in size by Dicer1. miRNAs then guide miRISC (RISC 
complex associated with the miRNA pathway), including the Agol protein, to degrade and/ or inhibit translation of (partially) complementary single-stranded (ss)RNAs (Asgari, 2014, 2015). However, our knowledge of the antiviral role of the miRNA pathway in mosquito-virus interactions is limited.

Arbovirus specific piRNAs of 25-29 nts length have also been reported in infected mosquitoes and mosquito-derived cells (Miesen et al., 2016; Varjak, Leggewie, et al., 2018). In Aedes aegypti-derived cells, virus-derived piRNA (vpiRNA) biogenesis is Piwi5/6 (depending on the investigated virus) and Ago3 dependent for Sindbis, Chikungunya and Dengue viruses (Miesen et al., 2015, 2016; Varjak, Dietrich, et al., 2018). The transcripts are bound by Ago3 (sense) and Piwi5/6 (antisense) and processed in the ping-pong amplification cycle. Resultant vpiRNAs have either a bias for uridine at position one or adenine at position 10 in the antisense and sense sequences, respectively (U1, A10) and a complementary region of 10 nucleotides (Miesen et al., 2015, 2016). In contrast, another Piwi protein, Piwi4, does not directly bind vpiRNAs of viral or transposon origin (Miesen et al., 2015) but preferentially binds antisense piRNAs derived from endogenous viral elements (EVEs). These EVEs can be integrated into the mosquito genome during RNA virus infection and act as an "adaptive immune response" combined with the produced vpiRNAs and Piwi4 (Tassetto et al., 2019). The knock-down of Piwi4 transcripts resulted in increased virus titer supporting its antiviral role. In contrast, the knock-down of piRNA pathway proteins did not have a strong antiviral role against the tested arboviruses so far (Schnettler et al., 2013; Varjak, Donald, et al., 2017; Varjak, Leggewie, et al., 2018; Varjak, Maringer, et al., 2017a); except for Rift Valley Fever virus (Dietrich, Jansen, et al., 2017). Piwi4 has been shown to interact with proteins of the piRNA and siRNA pathways; however, its antiviral activity against an arthropod-borne alphavirus is independent of $D c r 2$ activity in the Aag2 cells (Varjak, Maringer, et al., 2017a).

Compared to arboviruses, ISV interactions with the mosquito RNAi pathways are less studied. Studies on ISVs mainly focused on detection of virus specific small RNAs in persistently infected cell lines. Here, the production of vsiRNAs and, in some cases, vpiRNAs were detected for different families, including Flaviviridae, Birnaviridae and Phenuiviridae (Agboli et al., 2019; Frangeul et al., 2020; Öhlund et al., 2021) Our knowledge on RNAi-ISV interactions is further limited for insect-specific 
alphaviruses, as no persistently infected cell lines is known and (Blitvich \& Firth, 2015; Bolling et al., 2015) only four insect-specific alphaviruses have been identified in mosquitoes so far: Eilat virus (EILV), Taï Forest alphavirus (TALV), Mwinilunga alphavirus (MWAV) and Agua-Salud Alphavirus (ASALV) (Hermanns et al., 2017, 2020; Nasar et al., 2012; Torii et al., 2018). So far, only the latter has been studied for its interactions with the RNAi response (Hermanns et al., 2020). Indeed, ASALV infection in Aedes albopictus-derived cells induces the production of vsiRNAs, but lack the production of vpiRNAs. Moreover, it is unknown whether the siRNA pathway acts antiviral against ASALV.

In addition to the mosquitoes' ability to control virus replication through the RNAi pathways, viruses can also suppress the RNAi response. Indeed, some ISVs such as Culex Y virus and Mosinovirus, are known to interfere with the RNAi response (Fareh et al., 2018; Schuster et al., 2014; van Cleef et al., 2014) by encoding a RNAi suppressor protein. However, it is not known whether this is the case for insect-specific alphaviruses.

Here, we investigated the interactions of ASALV with the mosquito RNAi pathways in detail. We show the antiviral role of the exo-siRNA pathway against ASALV by using Ae. aegypti derived Dcr2 and Ago2 knock-out cell lines. ASALV-specific siRNAs were still produced in the absence of Ago2 but decreased in the Dcr2 knock-out cell line. ASALV triggered vpiRNA production through the pingpong production pathway only in Dcr2 knock-out cells. By knocking down additional key RNAi transcripts, we further show the involvement of Ago1, Ago2 and Piwi4 as antiviral against ASALV in Ae. aegypti derived cells.

\section{Methods}

\section{$\underline{\text { Cell lines }}$}

Aag2-AF5 (ECACC 19022601) is a single-cell clone of Aedes aegypti derived Aag2 cells. Aag2-AF319 (ECACC 19022602) is a Dcr2 knock-out (KO) cell line derived from AF5 cells (Varjak, Maringer, et al., 
2017b), and AF525 is an Ago2 knock-out cell line also derived from AF5 cells (Scherer et al., 2021).

Aedes albopictus derived C6/36 cells were used for virus production.

131 All cell lines were kept in Leibovitz's L15 Medium (ThermoFisher Scientific) supplemented with

$13210 \%$ tryptose phosphate broth (Gibco Life Technologies), $10 \%$ fetal bovine serum (ThermoFisher

133 Scientific), and $1 \%$ penicillin-streptomycin (ThermoFisher Scientific). All cell lines were grown at $13428^{\circ} \mathrm{C}$.

Previously isolated and plaque purified ASALV was used for all experiments (Hermanns et al., 2020).

Virus stocks were produced by inoculating C6/36 cells. The supernatant was harvested upon observation of morphological changes and was cleared from cell debris by centrifugation. For TCID50 virus quantification, $4 \times 10^{4} \mathrm{C} 6 / 36$ cells/ per well were seeded in 96-well plates 2 hours before purification using the MEGAscript RNAi kit (Thermo Fisher Scientific) according to the manufacturer's instructions. incubation, the infectious medium was replaced with $1 \mathrm{ml}$ of fresh L15 with supplements. Samples were taken at different time points $(0,24,48,72$ hours post-infection (hpi)). Infection and negative controls were performed in triplicates, and three independent experiments were performed. The 
with TRIzol LS (Invitrogen) according to manufacturer's protocol. QuantiTect SYBR Green qRT-

PCR one-step kit (Qiagen) was used to quantify ASALV with previously established primers

(Hermanns et al., 2020). Samples were run in technical triplicates. An in-run calibrator and an external standard curve were used to perform an absolute quantification using Roche Light Cycler 480 II.

\section{$\underline{\text { ASALV infection in knock-out cells and small RNA sequencing }}$}

$3 \times 10^{5}$ cells/well (AF5, AF525, AF319) were seeded in 24-well plates and infected with ASALV at

MOI 0.5 the following day. Total RNA of infected cells was isolated at $48 \mathrm{hpi}$ with Trizol according to manufacturer's protocol using glycogen as a carrier. QuantiTect SYBR Green qRT-PCR one-step kit (Qiagen) was used to quantify ASALV with previously established primers (Hermanns et al., 2020). ASALV RNA fold change was calculated using the $2^{-\Delta \Delta C T}$ method with Ribosomal protein S7 RNA as the housekeeping gene and AF5 cells as the control group.

To investigate the production of ASALV specific small RNAs in AF5, AF525 and AF319 cells, $8 \times 10^{5}$ cells were seeded in a 6-well plate and infected with ASALV (MOI 1). Total RNA was isolated at 48 hpi with TRIzol (Ambion), according to manufacturer's protocol with glycogen as a carrier. Small RNAs of $1 \mu \mathrm{g}$ total RNA were sequenced using BGISEQ-500 at BGI Tech (Hong Kong, China) as previously described (Scherer et al. 2021). For one of the AF525 samples (Figure S1), total RNA was sequenced at IKMB (Kiel, Germany), using $100 \mathrm{ng}$ total RNA for library preparation with the NEXTFLEX ${ }^{\circledR}$ Small RNA-Seq Kit v3 (PerkinElmer Inc., Waltham, MA, USA), followed by library sequencing on one lane NovaSeq6000 SP v1.0 (2x50bp). Data analyses were performed as previously described (Varjak, Dietrich, et al., 2018). The ASALV genome sequence was used as template (MK959115). Small RNA sequencing data is available in the NCBI Sequence Read Archive under BioProject ID PRJNA725665.

\section{Knock-down experiments}

$2.5 \times 10^{5}$ AF5 cells/well were seeded in 24-well plates the day before transfection with $200 \mathrm{ng}$ of gene-specific dsRNAs or control dsRNA (dsLacZ) per well, and transfected using 1 ul of Dharmafect2 
reagent (GE Dharmacon). For siRNA knock-downs in knock-out cells, $20 \mathrm{nM}$ of either Piwi4 specific siRNAs or control siRNA (Horizon Discovery) was transfected using $2 \mu 1$ Dharmafect2 reagent (GE and Ribosomal protein S7 RNA as the housekeeping gene transcript. Results were analysed using the $2^{-\Delta \Delta C T}$ method with LacZ dsRNA samples as control. All qPCR reactions were performed in technical triplicates.

To assess whether the presence of ASALV in cells could suppress the RNA silencing response, AF5

192 cells were seeded in 24-well plates $\left(1.8 \times 10^{5}\right.$ cell/well) one day prior to ASALV infection (MOI 10). protocol on a Glomax luminometer. test) of the data were tested. The student's t-test was used for normally distributed homoscedastic data, statistically significant. 


\section{Results}

208

\section{ASALV efficiently replicates in AF5 cells}

The successful replication of ASALV has been previously shown in Ae. albopictus derived C6/36 and cells (Figure 1) without any visible cytopathic effect.

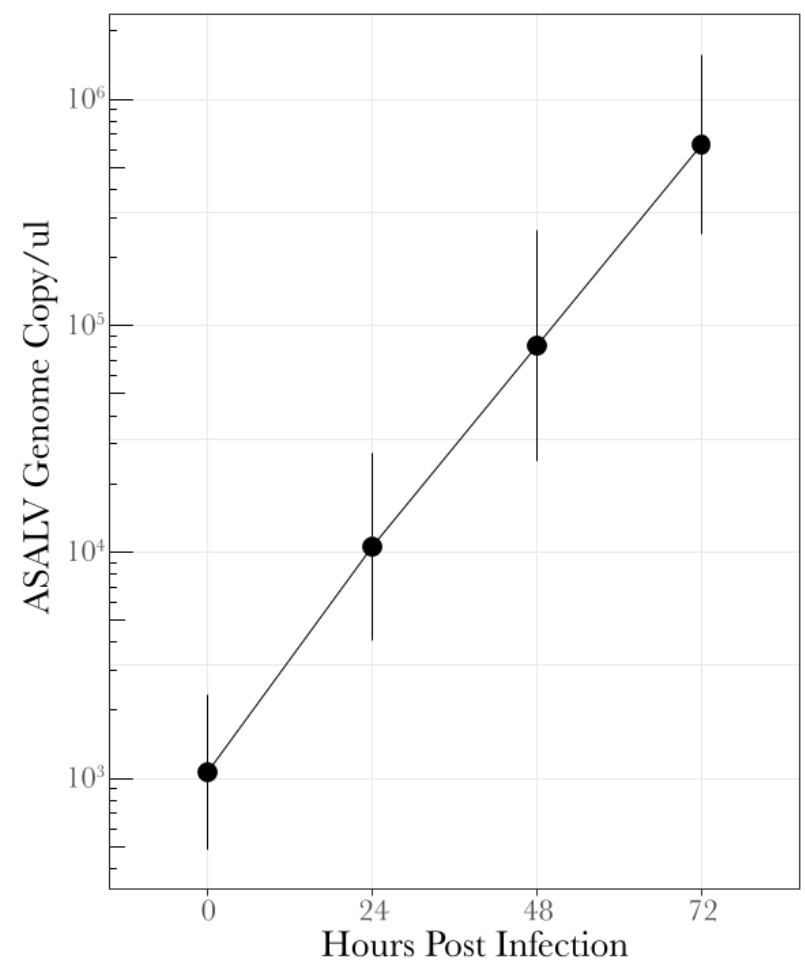

Figure 1: Growth Kinetics of ASALV in Aedes aegypti derived AF5 cells. AF5 cells were infected with ASALV with an MOI of 0.1. The supernatant was collected at different time points $(0,24,48$ and $72 \mathrm{hpi})$, and ASALV RNA was quantified by qRT-PCR. The average of three independent replicates (performed in triplicates) is shown with SEM. 
222 To investigate the effect of the siRNA pathway on ASALV replication, Dcr2 (AF319) and Ago2

223 (AF525) knock-out (KO) cells and control AF5 cells were infected with ASALV (MOI 0.5). ASALV

224 RNA fold change in the KO cells compared to AF5 cells at 48 hpi was quantified by qPCR. ASALV

225 RNA increased significantly in AF525 $(\mathrm{t}=5.2385, \mathrm{df}=7, \mathrm{p}=0.001)$ and AF319 cells $(\mathrm{t}=21.654, \mathrm{df}=$

$2267, \mathrm{p}<0.001$, Figure 2) compared to AF5 control cells.

227

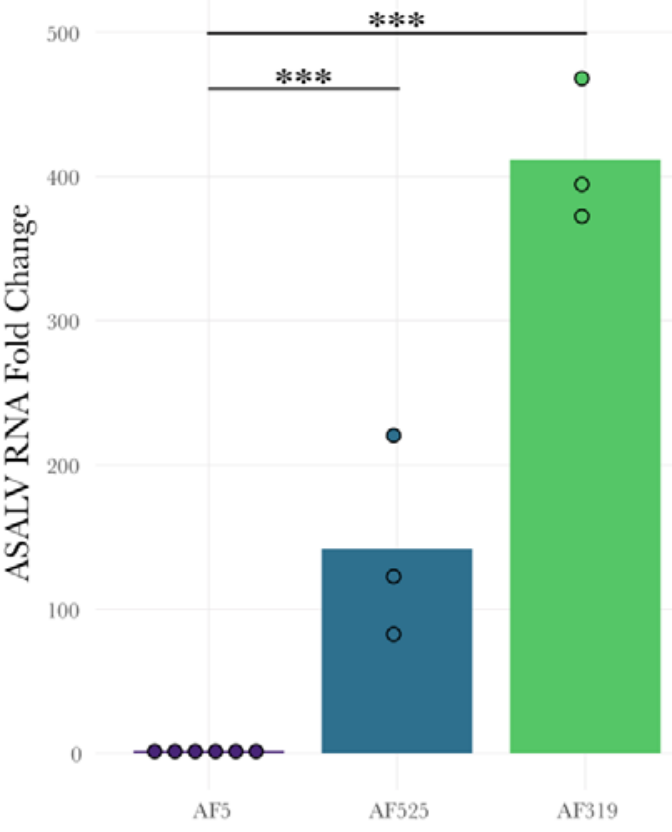

Figure 2: Increased ASALV replication in Dcr2 (AF319) and Ago2 (AF525) Ae. aegypti derived knock-out cells. AF319, AF525 and AF5 cells were infected with ASALV (MOI 0.5). ASALV RNA fold change in infected cells was quantified at $48 \mathrm{hpi}$, using the $2^{-\Delta \Delta \mathrm{CT}}$ method with Ribosomal protein S7 RNA as housekeeping gene and AF5 cells as control. Three independent replicates were performed for AF525, and AF319 cells $(n=3)$ and AF5 controls were repeated for each group $(n=6)$. Bar plots represent the mean of the replicates that were performed $(* * *: p<0.001)$.

\section{piRNA-sized small RNAs with ping-pong characteristics are only produced in Dcr2 KO cells}

To investigate the production of ASALV-specific small RNAs in the different cells, small RNA sequencing of ASALV infected cells was performed in Ae. aegypti-derived AF5, AF319 and AF525 cells. Cells were infected with ASALV (MOI 0.5), and total RNA was isolated at 48 hpi, followed by small RNA sequencing and bioinformatics analysis. Two independent replicates per cell line were performed, resulting in similar findings (Table 1 Figure 3, Figure S1-S2). 
bioRxiv preprint doi: https://doi.org/10.1101/2021.12.20.473494; this version posted December 20, 2021. The copyright holder for this preprint (which was not certified by peer review) is the author/funder, who has granted bioRxiv a license to display the preprint in perpetuity. It is made available under aCC-BY-NC-ND 4.0 International license.

\begin{tabular}{|c|c|c|c|c|c|c|}
\hline & \multicolumn{3}{|c|}{ Total reads } & \multicolumn{3}{|c|}{ ASALV-specific reads } \\
\hline Cell line & Total & $\begin{array}{l}21 \mathrm{nts} \\
\mathrm{rpm}^{*}\end{array}$ & $\begin{array}{l}27-28 \mathrm{nts} \\
\mathrm{rpm} *\end{array}$ & Total & $\begin{array}{l}\text { Proportion of } \\
21 \text { nts to } \\
\text { ASALV- } \\
\text { specific reads }\end{array}$ & $\begin{array}{l}\text { Proportion of } \\
27-28 \mathrm{nts} \\
\text { to ASALV- } \\
\text { specific reads }\end{array}$ \\
\hline AF5 (Figure 3) & $28,193,638$ & 9,996 & 215 & 361,122 & 0.780 & 0.017 \\
\hline AF5 (Figure S1) & $28,129,604$ & 2,581 & 113 & 97,986 & 0.741 & 0.033 \\
\hline AF525 (Figure 3) & $22,393,006$ & 147,349 & 1,308 & $4,284,285$ & 0.770 & 0.007 \\
\hline AF525 (Figure S1) & $70,276,976$ & 108,396 & 490 & $12,543,700$ & 0.607 & 0.003 \\
\hline AF319 (Figure 3) & $27,567,645$ & 1,234 & 1,859 & 254,288 & 0.134 & 0.202 \\
\hline AF319 (Figure S1) & $28,463,344$ & 1,713 & 2,148 & 284,136 & 0.172 & 0.215 \\
\hline
\end{tabular}

$242 *$ rpm $=$ reads per million 
$\mathbf{A}$

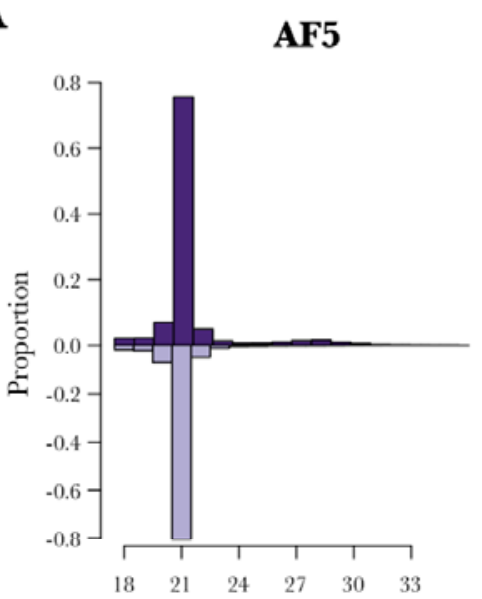

B

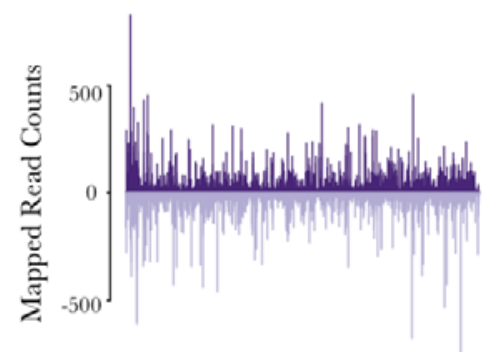

G

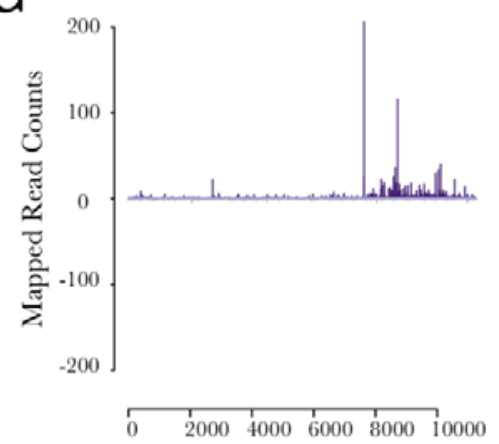

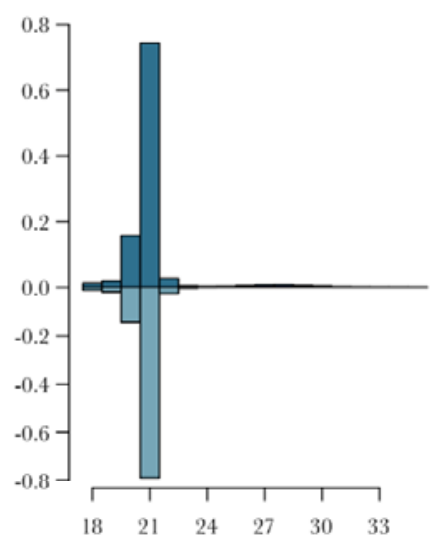

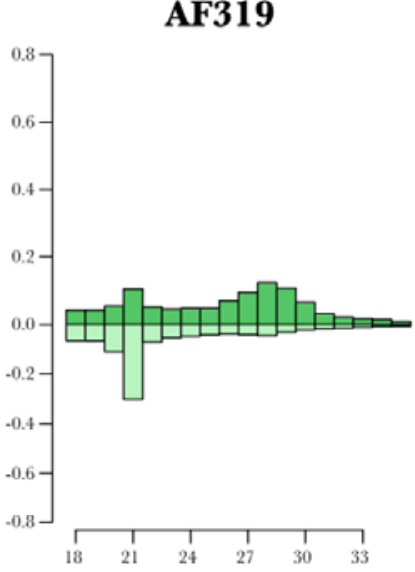

Read Length (nt)
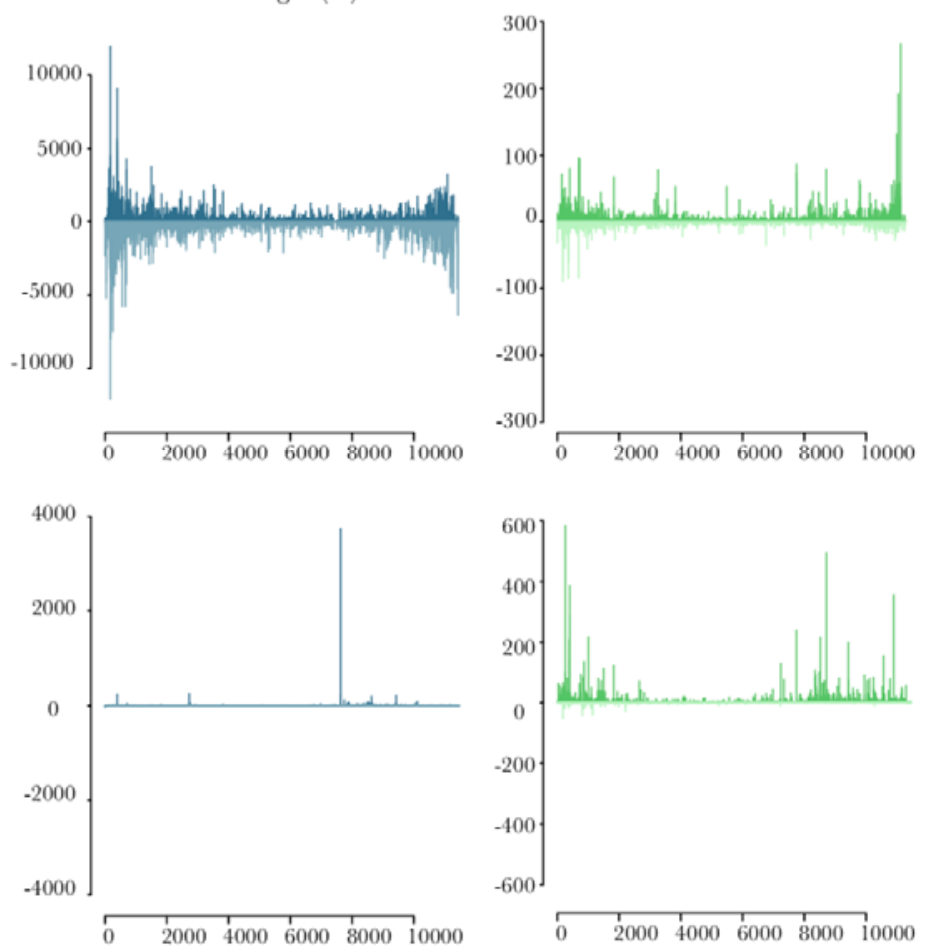

Genome Coordinates (nt)

Figure 3: ASALV-specific small RNA production in Ae. aegypti derived AF5, AF319 (Dcr2 KO) and AF525 (Ago2

KO). Cells were infected with ASALV (MOI 0.5). Total RNA was isolated at 48 hpi from the cells, small RNAs (18-40 nt)

were sequenced and mapped to the ASALV genome (sense, positive numbers) and antigenome (antisense, negative 
252 In AF5 cells, ASALV-specific siRNAs (Figure 3A) are produced and mapped across the genome

253 (sense) and antigenome (antisense, Figure 3B), similar to the results previously observed in U4.4 cells

254 (Hermanns et al., 2020). Similarly, in Ago2 KO AF525 cells, the majority of ASALV-specific small

255 RNAs are $21 \mathrm{nts}$ long vsiRNAs (Figure 3A). They also map across the whole genome and antigenome,

256 although with a bias to the 5'and 3'end (Figure 3B); which is not observed in AF5 cells. In Dcr2 KO

257 AF319, ASALV-specific siRNAs are strongly decreased, and a majority of them map to the 3'end of 258 the ASALV genome.

$\mathbf{A}$

AF5

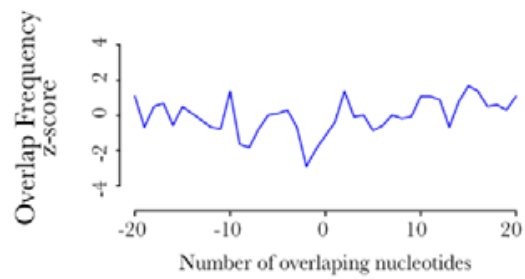

B

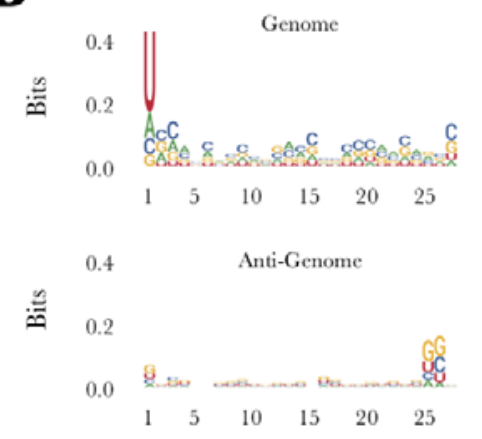

AF525
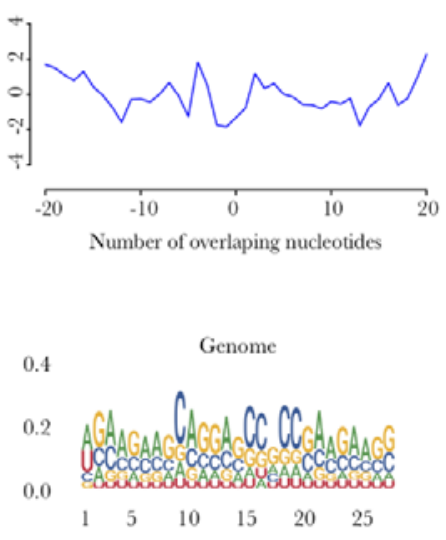

$0.4 \quad$ Anti-Genome

0.2

$\begin{array}{llllllll}0.0 & 9 & -1 & & & & & \\ 1 & 5 & 10 & 15 & 20 & 25\end{array}$
AF319
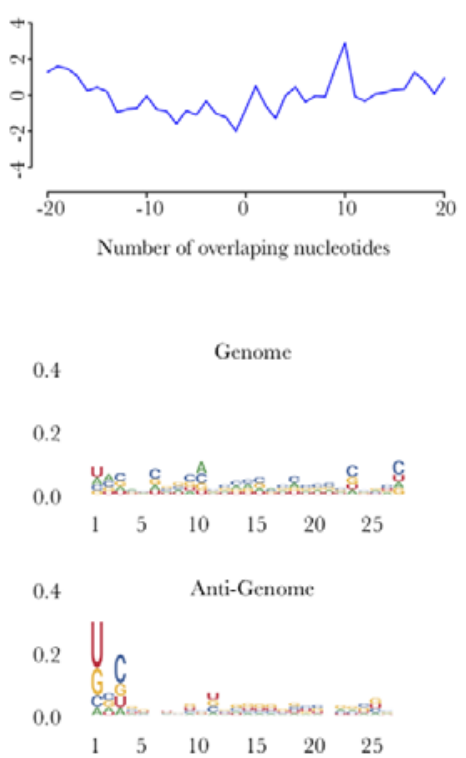

Figure 4: Characterization of ASALV specific 25-29 nts long small RNAs in Ae. aegypti-derived AF5, AF319 (Dcr2

260

261

262

263

264

265

266

267

KO) and AF525 (Ago2 KO). A. Overlap frequency of sense and antisense 25-29 nts long ASALV- specific small RNAs was calculated. B. Logo sequence plots show the sequence bias in various positions of $27 \mathrm{nts}$ (as representative of vpiRNAs) long ASALV-specific small RNAs for genomic (upper panel) and antigenomic (lower panel) small RNAs. The figure is a representative result of two independent experiments.

piRNA-sized small RNAs were observed at a low concentration in both AF5 and AF525 cells (Figure 3A-C) and did not show the "ping-pong" amplification characteristics (Figure 4). In contrast, AF319 cells produce ASALV-specific piRNA-sized small RNAs (Figure 3A-C) with the ping-pong amplification characteristics (Figure 4). Antisense and sense piRNA-sized small RNAs showed a clear 
271 piRNA-sized small RNA sequence, although the bias was not very strong. In antisense piRNA-sized the subgenomic RNA, encoding for the capsid protein, similar to vpiRNAs produced by arthropodborne alphaviruses (Miesen et al., 2015; Schnettler et al., 2013). However, in AF319 cells, some piRNA-sized small RNAs map also to the $5{ }^{`}$ of the genome (Figure 4C).

Taken together, vsiRNAs are the main small RNA species produced against ASALV infection under normal circumstances. In the absence of $D c r 2$, ASALV can induce piRNA-sized small RNAs with sequence characteristics indicative of the ping-pong amplification pathway.

Increased ASALV infection in the knock-out cell lines supports the involvement of the siRNA pathway in the antiviral defense against ASALV. To investigate the involvement of the other RNAi pathway proteins against ASALV in Ae. aegypti-derived AF5 cells, transcripts of different RNAi proteins were silenced by transfecting cells with sequence-specific dsRNAs (Agol, Ago2, Ago3, Piwi4, Piwi5, Piwi6), prior to ASALV infection (MOI 0.5, Figure 5A). compared to control cells (transfected with dsRNA specific to LacZ). Viral replication increased significantly in cells where Agol $(\mathrm{t}=2.817, \mathrm{df}=4.665, \mathrm{p}=0.040), \operatorname{Ago} 2(\mathrm{t}=6.437, \mathrm{df}=5.039, \mathrm{p}=$ 0.001) or Piwi4 $(\mathrm{t}=2.628, \mathrm{df}=8.543, \mathrm{p}=0.029)$ transcripts were silenced (Figure 5B). The ASALV RNA fold change was more pronounced in Ago2 silenced cells than in Agol and Piwi4 silenced cells

292 (Table S2). Furthermore, when Piwi4 the silencing was conducted using Piwi4 siRNAs, instead of 
$\mathbf{A}$

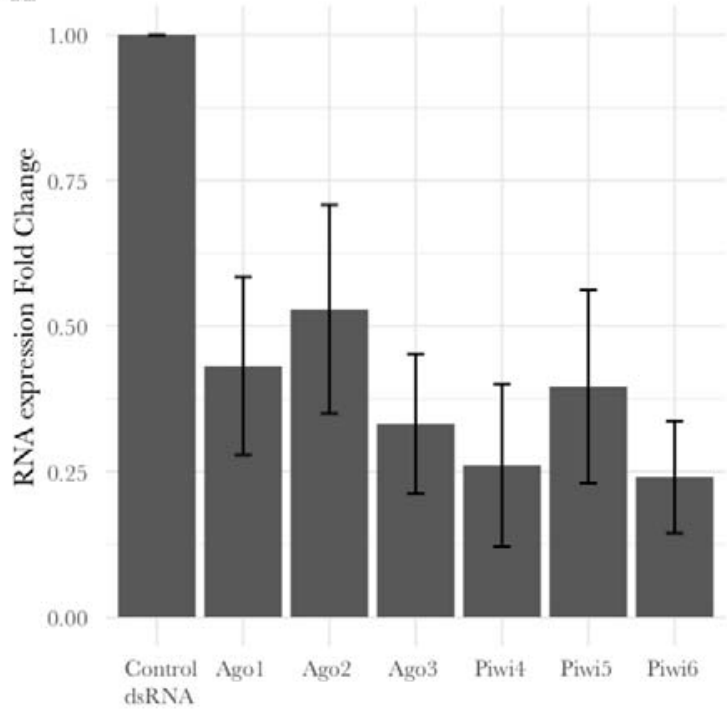

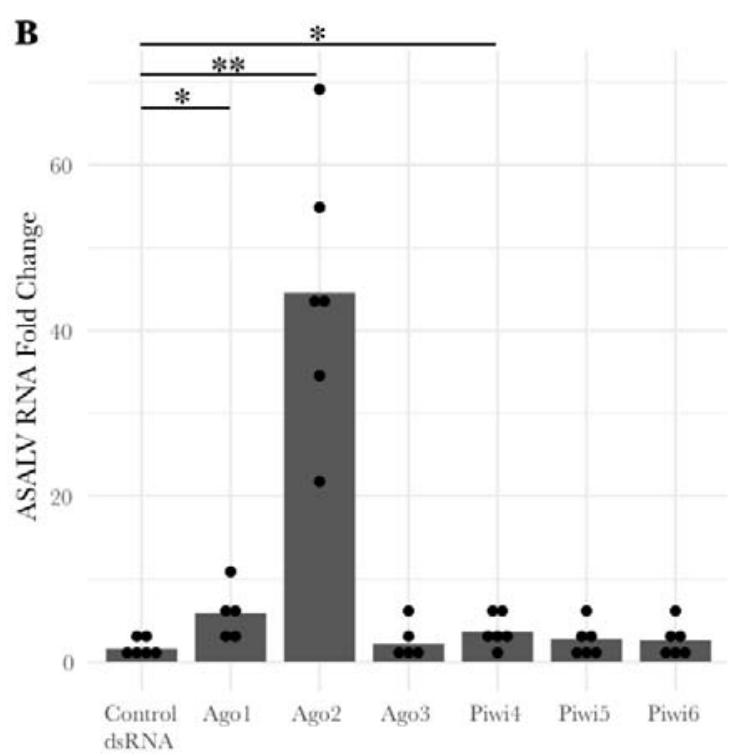

295

296

297

298

299

300

301

302

303

304

305

306

Figure 5: Ago1, Ago2 and Piwi4 silencing increases ASALV replication in Ae.. aegypti derived AF5 cells. Cells were transfected either with gene-specific dsRNAs or control dsRNA (LacZ-specific). The following day, cells were infected with ASALV (MOI 0.5), and total RNA was isolated $48 \mathrm{~h}$ post-infection. A. mRNA targets were quantified using gene-specific primers and Ribosomal protein S7 RNA as housekeeping transcript. $2^{-\Delta \Delta}$ of of mRNA targets was calculated with the mean normalised RNA expression of a given transcript in the control cells, within the same replicate, as control. The resulting mean fold change and standard error of the mean are shown. B. ASALV RNA was quantified using ASALV specific primers and Ribosomal protein S7 RNA as housekeeping transcript. ASALV RNA fold change was calculated using $2^{-\Delta \Delta C T}$ method with the mean of normalised expression of ASALV RNA, of all replicates, in the control cells as control. Bar plots represent the mean fold change for each group calculated. At least five independent replicates were performed. $(*: \mathrm{p}=<0.05, * *$ : $\mathrm{p}<0.01)$.

\section{No RNAi suppressor effect of ASALV was detected in AF5 cells}

Several insect viruses have been reported to encode proteins that interfere with the antiviral RNAi pathway, named viral suppressors of RNAi (VSR). VSRs can interfere at different steps of the RNAi pathways by interacting with key molecules (e.g. dsRNA or siRNAs) or proteins (e.g. Ago2, Dcr2), mostly of the exo-siRNA pathway. To determine if ASALV can suppress the exo-siRNA response in mosquito-derived cells, a previously used luciferase-based RNAi suppressor assay was performed (Ongus et al., 2006; Varjak, Maringer, et al., 2017). AF5 cells were either infected with ASALV (MOI 10) or mock-infected. After 24 hpi, cells were co-transfected with Firefly and Renilla luciferase (internal control) expression constructs as well as dsRNA (FFluc or LacZ as control) or siRNA (siFFluc, siHyg as control) to induce silencing. Luciferase activity was measured 24 hpi and sequencespecific silencing of Firefly luciferase in ASALV or mock-infected cells were compared. 
317 Relative luciferase activity was significantly reduced in cells transfected with FFluc dsRNA compared

318 to controls in both ASALV $(\mathrm{t}=-12.785, \mathrm{df}=2, \mathrm{p}=0.006$; Figure $6 \mathrm{~A})$ and mock-infected cells $(\mathrm{t}=-$

$31965.212, \mathrm{df}=2, \mathrm{p}<0.001$; Figure 6A). Similarly, luciferase expression was significantly silenced when

320 ASALV-infected $(\mathrm{t}=-15.469, \mathrm{df}=2, \mathrm{p}=0.004)$ or mock -infected cells $(\mathrm{t}=-20.322, \mathrm{df}=2, \mathrm{p}=0.002)$

321 were transfected with siFFluc compared to control siHyg transfection (Figure 6B). No difference in

322 silencing of luciferase could be observed between mock or ASALV infected cells whether the

323 silencing was induced by dsRNA $(t=0.281, d f=2.160, p=0.803$; Figure6A $)$ or siRNA $(t=0.881$, df

$324=4, p=0.428$; Figure $6 B$ ). Hence in our experimental setting, we did not detect any significant RNAi

325 suppressor activity of ASALV in AF5 cells.
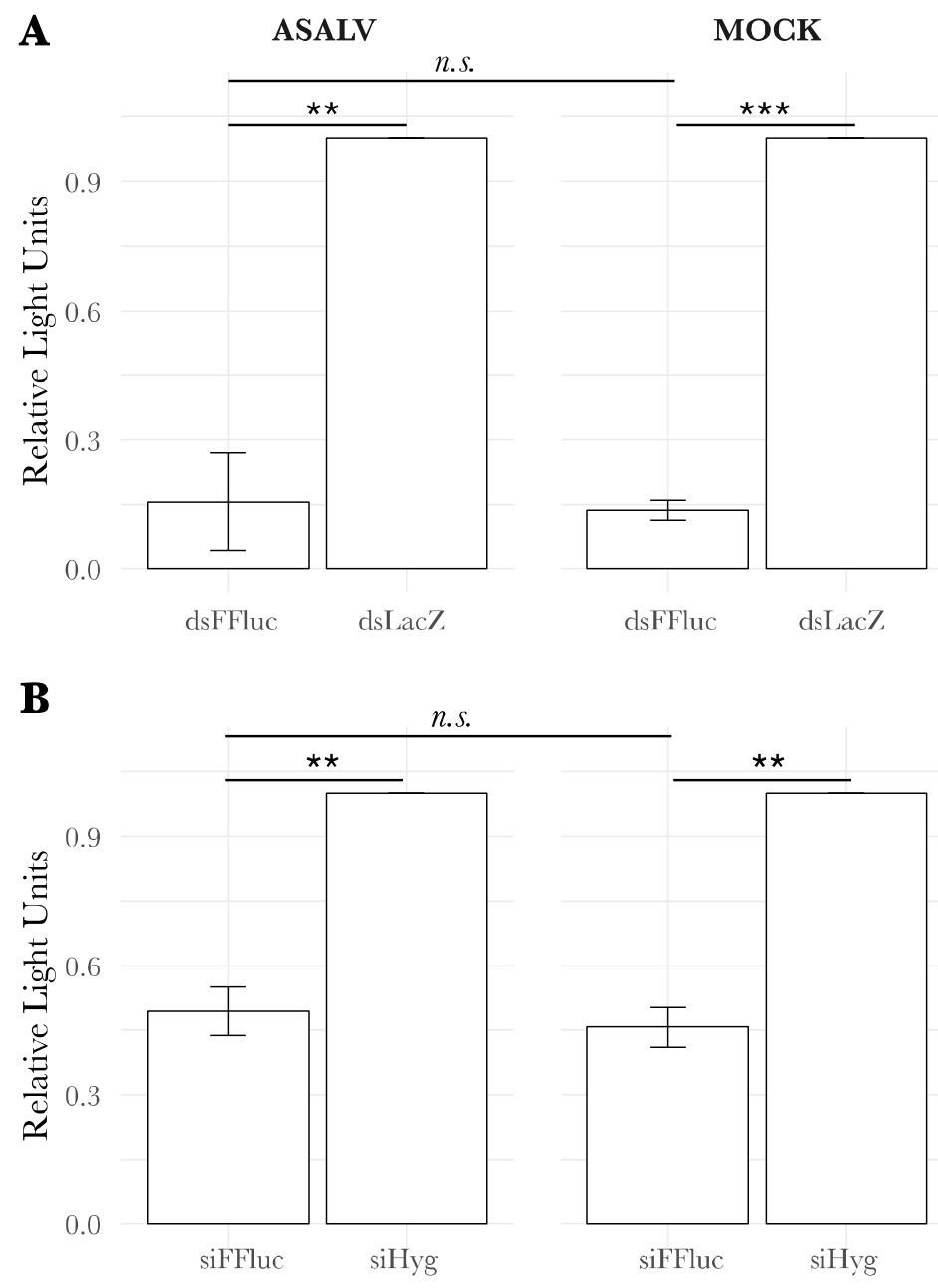

Figure 6: No RNAi suppressor effect of ASALV was detected in AF5 cells. AF5 cells are either mock-infected (cell 
culture media) or infected with ASALV (MOI 10). Following, cells were transfected with Firefly (FFluc) and Renilla luciferase (Rluc) expression constructs and either with $0.5 \mathrm{ng}$ dsRNA (A) or $0.1 \mathrm{ng}$ siRNA (B). Luciferase was measured using the Dual luciferase assay and FFluc expression was normalised to Rluc as an internal control (relative light units). FFluc/Rluc expression in the dsRNA(dsFluc) or siRNA(siFluc) transfected cells were normalised to control transfected cells (dsLacZ or siHyg). The mean of three independent experiments in triplicates are shown with SEM (***: p<0.001, **: p<0.01, n.s.: not significant).

\section{Discussion}

RNA interference (RNAi) is an important antiviral response in insects, including mosquitoes. The interaction between the mosquito RNAi pathways and a variety of viruses can be identified by detecting virus-specific small RNAs and increased viral infection in case of silencing of key proteins of the different RNAi pathways. RNAi has been shown to act antiviral in mosquitoes against all tested viruses so far, although differences regarding the importance of specific pathways or proteins have been reported (Liu et al., 2019). Our knowledge about the antiviral RNAi response in mosquitoes comes from arbovirus studies, although mosquitoes often harbour insect-specific viruses (ISVs). Small RNAs specific to a variety of ISVs were found in infected cells and mosquitoes. However, the antiviral role of the RNAi pathway against ISVs is not known (Agboli et al., 2019). Here we identified the antiviral function of the mosquito RNAi pathways against an insect-specific alphavirus for the first time.

The only previous study investigating an RNAi response specific to an insect-specific alphavirus showed the production of ASALV-specific 21 nts vsiRNAs in Ae. albopictus derived (U4.4) cells; although no vpiRNAs was observed (Hermanns et al., 2020). Our results confirm this previously reported lack of ASALV-specific piRNA production in Ae. aegypti-derived RNAi competent AF5 cells (Figure 4). In contrast, arthropod-borne alphaviruses induce both vsiRNAs and vpiRNAs in vitro in Ae. aegypti and Ae. albopictus-derived cell lines, as well as in mosquitoes (Cirimotich et al., 2009; Goic et al., 2016; Morazzani et al., 2012; Schnettler et al., 2013; Siu et al., 2011a; Vodovar et al., 2012). Despite the difference in the small RNAs that are produced during infection, the mapping of 
ASALV specific siRNAs (both in AF5 and U4.4 cells) was very similar to the mapping of arthropodand hot spots (Morazzani et al., 2012; Schnettler et al., 2013; Siu et al., 2011b). This suggests that similar to arthropod-borne alphaviruses, ASALV also mainly induces vsiRNA production through dsRNA replicative intermediates.

ASALV replication is increased in both Ago2 silenced (Figure 5B, Table S2) and Ago2 or Dcr2 knock-out cells (Figure 2); highlighting the antiviral role of the exo-siRNA pathway against ASALV. Similarly, silencing or knock-out of $A g o 2$ or $D c r 2$ induced an increase in infection of tested arthropodborne alphaviruses (Campbell et al., 2008; Schnettler et al., 2013; Sucupira et al., 2020; Varjak, Dietrich, et al., 2018; Varjak, Donald, et al., 2017). Furthermore, similar results have been found for arboviruses belonging to other virus families or orders (Liu et al., 2019), except for ZIKV, where no antiviral activity was reported for Ago2 (Scherer et al., 2021; Varjak, Donald, et al., 2017). For the arthropod-borne alphavirus SFV, the magnitude of increase in infection was similar in Dcr2 and Ago2 knock-out cells (Scherer et al., 2021). In contrast, for ASALV, the differences between Ago2 and Dcr2 knock-out cells suggest an additional role of $D c r 2$ in the antiviral response against ASALV independent of $A g o 2$. For instance, Dcr2 can detect viral RNA and induce an antiviral protein, Vago, which activates the Jak-STAT pathway leading to an antiviral effect in Culex quinquefasciatus (Hsu)derived cells (Paradkar et al., 2012, 2014). Notably, however, Vago does not seem to be induced in infected Ae. aegypti-derived Aag2 cells (Russell et al., 2021). Alternatively, this increased antiviral effect of $D c r 2$ against ASALV might be linked to another yet unknown antiviral pathway related to Dcr2 activity.

ASALV-specific piRNA-sized small RNAs with ping-pong amplification characteristics were produced only in $D c r 2$ knock-out cells. Previous reports have also shown an increase of SFV-specific vpiRNAs in cells lacking the Dcr2 protein (Varjak, Maringer, et al., 2017b). It is possible that the increase in the vpiRNA production is a result of (i) the increased viral replication due to the lack of the antiviral $D c r 2$ protein, (ii) the high concentration of ASALV RNA in the cytoplasm that is not cut into vsiRNAs or (iii) a combination of both. Although ASALV replication was increased in Ago2 knock- 
out cells, no ping-pong specific vpiRNAs were detected. While this could mean that increased viral replication is not solely sufficient for ASALV specific vpiRNA production, it has to be noted that the increase in ASALV replication in Ago2 knock-out cells was still lower compared to Dcr2 knock-out cells. Therefore, it could be that the increased ASALV RNA concentration in Ago2 KO cells is not sufficient to trigger vpiRNA production, in contrast to $D c r 2 \mathrm{KO}$ cells. In addition, it is likely that in Dcr2 knock-out cells specifically, the amount of viral dsRNA molecules would increase. As the precise trigger for vpiRNA production in mosquitoes is not yet known, it could be that the concentration of ASALV dsRNA in Dcr2 knock-out cells could play a role in triggering vpiRNA production. On the other hand, the putative essential proteins for the biogenesis of vpiRNAs, Piwi5 and Ago3, were not antiviral against ASALV (Figure 5B), consistent with findings from arthropodborne alphaviruses(Miesen et al., 2015; Varjak, Dietrich, et al., 2018; Varjak, Donald, et al., 2017). the production of SFV- or SINV- specific vpiRNAs, but it has recently been shown to bind DENVspecific piRNAs derived from viral cDNA in infected Ae. aegypti (Tassetto et al., 2019). While an interaction between Piwi4 and piRNA as well as siRNA pathway proteins, including Dcr2, has previously been shown, Piwi4 antiviral activity is independent of Dcr2 in SFV infected cells (Joosten et al., 2021; Varjak, Maringer, et al., 2017a). To check this for ASALV, we silenced Piwi4 by adding siRNAs, both in Dcr2 competent and knock-out cell lines. While the silencing of Piwi4 through siRNA increased ASALV replication, the increase was not significant in either of the cell lines (Figure S3). If this is due to the slightly lower silencing efficiency with siRNAs compared to dsRNA is not 
411 Schnettler et al., 2013). Similar increases in virus infection upon Ago1 silencing have been reported

412 for midge-borne orthobunyaviruses in Ae. aegypti derived cells in contrast to mosquito-borne

413 orthobunyaviruses (Dietrich, Shi, et al., 2017). Additional experiments are needed to determine if the

414 difference in Agol activity against arthropod-borne alphaviruses compared to insect-specific

415 alphaviruses can be generalised.

416 Many viruses infecting insects encode proteins to suppress the RNAi pathway, such as Flock House

417 Virus or Culex Y virus (O'Neal et al., 2014). Several arboviruses, such as Dengue and West Nile

418 Virus, have also been shown to interfere with the RNAi response by employing competitive substrates

419 for Dcr2 derived from their nucleic acids (O'Neal et al., 2014). Furthermore, recent work has identified

420 the non-structural protein NS2A of flaviviruses as a potent suppressor of RNAi (Qiu et al., 2020). In

421 our experimental system, we did not observe any RNAi suppressor activity of ASALV.

422 ISVs belonging to some of the arbovirus families and orders, such as Bunyavirales (Marklewitz et al.,

423 2015) and Flaviviridae (Cook et al., 2019), are thought to be ancestral to arboviruses, suggesting that

424 dual-host (invertebrate-vertebrate) tropism evolved from invertebrate specific viruses. As not many

425 insect-specific alphaviruses have been discovered so far, it is difficult to identify whether insect-

426 specific viruses or the arthropod-borne alphaviruses are the ancestors in the alphavirus genus (Halbach

427 et al., 2017). Nevertheless, like other insect-specific alphaviruses so far, ASALV is basal to the

428 Western Equine encephalitis virus complex clade, suggesting arthropod-borne alphaviruses in this

429 clade could have evolved from an ancestral insect-specific virus (Halbach et al., 2017; Hermanns et

430 al., 2020). It is also possible that the changes in the mosquito-virus interactions drive their evolution

431 resulting in their ability to transmit to vertebrates. In this context, differences between arboviral and

432 insect-specific alphaviruses' interaction with mosquito RNAi pathways could be one of the reasons

433 why ISVs were restricted to invertebrate hosts. In contrast to arthropod-borne alphaviruses studied so

434 far, we showed that ASALV specific vpiRNAs are not produced in Dcr2 competent cells, and Agol

435 was antiviral against ASALV. However, to be able to generalise this observation to other insect-

436 specific alphaviruses, more studies describing their interactions with mosquito hosts are needed.

437 Further studies taking both the persistent nature of ISVs and the tissue-specificity of the RNAi 
438

439

440

441

442

443

444

445

446

447

448

449

450

451

452

453

454

455

456

457

458

459

460

461

462

463

464

465

466

467

468

469

470

471

472

response into account could determine whether the interactions of insect-specific alphaviruses with the RNAi pathways restrict ISVs to their mosquito hosts.

\section{Funding}

This research was supported by the Deutsche Forschungszentrum für Infektionszforschung (DZIF) and the German Federal Ministry of Food and Agriculture (BMEL) through the Federal Office for Agriculture and Food (BLE), grant numbers 2819113919.

The funders had no role in the design of the study, in the collection, analyses, or interpretation of data, in the writing of the manuscript, or in the decision to publish the results.

\section{References}

Agboli, E., Leggewie, M., Altinli, M., \& Schnettler, E. (2019). Mosquito-Specific Viruses-Transmission and Interaction. Viruses, 11(9), 873. https://doi.org/10.3390/v11090873

Altinli, M., Schnettler, E., \& Sicard, M. (2021). Symbiotic Interactions Between Mosquitoes and Mosquito Viruses. Frontiers in Cellular and Infection Microbiology, 11(August), 1-14. https://doi.org/10.3389/fcimb.2021.694020

Asgari, S. (2014). Role of microRNAs in vrbovirus/vector interactions. Viruses, 6(9), 3514-3534. https://doi.org/10.3390/v6093514

Asgari, S. (2015). Regulatory role of cellular and viral microRNAs in insect-virus interactions. Current Opinion in Insect Science, 8, 104-110. https://doi.org/10.1016/j.cois.2014.12.008

Atoni, E., Zhao, L., Karungu, S., Obanda, V., Agwanda, B., Xia, H., \& Yuan, Z. (2019). The discovery and global distribution of novel mosquito-associated viruses in the last decade (2007-2017). Reviews in Medical Virology, 29(6). https://doi.org/10.1002/rmv.2079

Blitvich, B. J., \& Firth, A. E. (2015). Insect-specific flaviviruses: A systematic review of their discovery, host range, mode of transmission, superinfection exclusion potential and genomic organization. In Viruses (Vol. 7, Issue 4, pp. 1927-1959). https://doi.org/10.3390/v7041927

Bolling, B., Weaver, S., Tesh, R., \& Vasilakis, N. (2015). Insect-Specific Virus Discovery: Significance for the Arbovirus Community. Viruses, 7(9), 4911-4928. https://doi.org/10.3390/v7092851

Bronkhorst, A. W., \& Van Rij, R. P. (2014). The long and short of antiviral defense: Small RNA-based immunity in insects. Current Opinion in Virology, 7(1), 19-28.

https://doi.org/10.1016/j.coviro.2014.03.010

Campbell, C. L., Keene, K. M., Brackney, D. E., Olson, K. E., Blair, C. D., Wilusz, J., \& Foy, B. D. (2008). Aedes aegypti uses RNA interference in defense against Sindbis virus infection. $B M C$ Microbiology, 8, 1-12. https://doi.org/10.1186/1471-2180-8-47 
473

474

475

476

477

478

479

480

481

482

483

484

485

486

487

488

489

490

491

492

493

494

495

496

497

498

499

500

501

502

503

504

505

506

507

508

509

510

511

512

513

Carissimo, G., Pondeville, E., McFarlane, M., Dietrich, I., Mitri, C., Bischoff, E., Antoniewski, C., Bourgouin, C., Failloux, A.-B., Kohl, A., \& Vernick, K. D. (2015). Antiviral immunity of Anopheles gambiae is highly compartmentalized, with distinct roles for RNA interference and gut microbiota. Proceedings of the National Academy of Sciences, 112(2), E176-E185. https://doi.org/10.1073/pnas.1412984112

Cirimotich, C. M., Scott, J. C., Phillips, A. T., Geiss, B. J., \& Olson, K. E. (2009). Suppression of RNA interference increases alphavirus replication and virus-associated mortality in Aedes aegypti mosquitoes. BMC Microbiology, 9, 1-13. https://doi.org/10.1186/1471-2180-9-49

Cook, S., Moureau, G., Kitchen, A., Gould, E. A., Lamballerie, X. De, Holmes, E. C., \& Harbach, R. E. (2019). Molecular evolution of the insect-specific flaviviruses. May, 223-234. https://doi.org/10.1099/vir.0.036525-0

Dietrich, I., Jansen, S., Fall, G., Lorenzen, S., Rudolf, M., Huber, K., Heitmann, A., Schicht, S., Elliott, R. M., Diallo, M., Sall, A. A., \& Failloux, A. (2017). RNA Interference Restricts Rift Valley. MSphere, 2(3), 1-17. https://doi.org/10.1128/mSphere.00090-17

Dietrich, I., Shi, X., McFarlane, M., Watson, M., Blomström, A. L., Skelton, J. K., Kohl, A., Elliott, R. M., \& Schnettler, E. (2017). The Antiviral RNAi Response in Vector and Non-vector Cells against Orthobunyaviruses. PLoS Neglected Tropical Diseases, 11(1), 1-18. https://doi.org/10.1371/journal.pntd.0005272

Donald, C. L., Kohl, A., \& Schnettler, E. (2012). New insights into control of arbovirus replication and spread by insect RNA interference pathways. Insects, 3(2), 511-531. https://doi.org/10.3390/insects3020511

Fareh, M., van Lopik, J., Katechis, I., Bronkhorst, A. W., Haagsma, A. C., van Rij, R. P., \& Joo, C. (2018). Viral suppressors of RNAi employ a rapid screening mode to discriminate viral RNA from cellular small RNA. Nucleic Acids Research, 46(6), 3187-3197. https://doi.org/10.1093/nar/gkx1316

Frangeul, L., Blanc, H., Saleh, M. C., \& Suzuki, Y. (2020). Differential small RNA responses against coinfecting insect-specific viruses in aedes albopictus mosquitoes. Viruses, 12(4). https://doi.org/10.3390/v12040468

Goic, B., Stapleford, K. A., Frangeul, L., Doucet, A. J., Gausson, V., Blanc, H., Schemmel-Jofre, N., Cristofari, G., Lambrechts, L., Vignuzzi, M., \& Saleh, M.-C. (2016). Virus-derived DNA drives mosquito vector tolerance to arboviral infection. Nature Communications, 7(1), 12410. https://doi.org/10.1038/ncomms12410

Halbach, R., Junglen, S., \& van Rij, R. P. (2017). Mosquito-specific and mosquito-borne viruses: evolution, infection, and host defense. Current Opinion in Insect Science, 22, 16-27. https://doi.org/10.1016/j.cois.2017.05.004

Hermanns, K., Marklewitz, M., Zirkel, F., Overheul, G. J., Page, R. A., Loaiza, J. R., Drosten, C., Van Rij, R. P., \& Junglen, S. (2020). Agua salud alphavirus defines a novel lineage of insect-specific alphaviruses discovered in the New World. Journal of General Virology, 101(1), 96-104. https://doi.org/10.1099/JGV.0.001344

Hermanns, K., Zirkel, F., Kopp, A., Marklewitz, M., Rwego, I. B., Estrada, A., Gillespie, T. R., Drosten, C., \& Junglen, S. (2017). Discovery of a novel alphavirus related to Eilat virus. Journal of General Virology, 98(1), 43-49. https://doi.org/10.1099/jgv.0.000694 
514

Joosten, J., Taşköprü, E., Jansen, P. W. T. C., Pennings, B., Vermeulen, M., \& van Rij, R. P. (2021). PIWI proteomics identifies Atari and Pasilla as piRNA biogenesis factors in Aedes mosquitoes. Cell Reports, 35(5). https://doi.org/10.1016/j.celrep.2021.109073

Keene, K. M., Foy, B. D., Sanchez-Vargas, I., Beaty, B. J., Blair, C. D., \& Olson, K. E. (2004). RNA interference acts as a natural antiviral response to O'nyong-nyong virus (Alphavirus; Togaviridae) infection of Anopheles gambiae. Proceedings of the National Academy of Sciences, 101(49), 17240-17245. https://doi.org/10.1073/pnas.0406983101

Leggewie, M., \& Schnettler, E. (2018). RNAi-mediated antiviral immunity in insects and their possible application. Current Opinion in Virology, 32, 108-114. https://doi.org/10.1016/j.coviro.2018.10.004

Liu, J., Swevers, L., Kolliopoulou, A., \& Smagghe, G. (2019). Arboviruses and the challenge to establish systemic and persistent infections in competent mosquito vectors: The interaction with the RNAi mechanism. Frontiers in Physiology, 10(JUL), 1-29. https://doi.org/10.3389/fphys.2019.00890

Marklewitz, M., Zirkel, F., Kurth, A., Drosten, C., \& Junglen, S. (2015). Evolutionary and phenotypic analysis of live virus isolates suggests arthropod origin of a pathogenic RNA virus family. Proceedings of the National Academy of Sciences, 112(24), 7536-7541. https://doi.org/10.1073/pnas.1502036112

McFarlane, M., Arias-Goeta, C., Martin, E., O'Hara, Z., Lulla, A., Mousson, L., Rainey, S. M., Misbah, S., Schnettler, E., Donald, C. L., Merits, A., Kohl, A., \& Failloux, A. B. (2014). Characterization of Aedes aegypti Innate-Immune Pathways that Limit Chikungunya Virus Replication. PLoS Neglected Tropical Diseases, 8(7). https://doi.org/10.1371/journal.pntd.0002994

Miesen, P., Girardi, E., \& Van Rij, R. P. (2015). Distinct sets of PIWI proteins produce arbovirus and transposon-derived piRNAs in Aedes aegypti mosquito cells. Nucleic Acids Research, 43(13), 6545-6556. https://doi.org/10.1093/nar/gkv590

Miesen, P., Joosten, J., \& van Rij, R. P. (2016). PIWIs Go Viral: Arbovirus-Derived piRNAs in Vector Mosquitoes. PLoS Pathogens, 12(12), 1-17. https://doi.org/10.1371/journal.ppat.1006017

Morazzani, E. M., Wiley, M. R., Murreddu, M. G., Adelman, Z. N., \& Myles, K. M. (2012). Production of virus-derived ping-pong-dependent piRNA-like small RNAs in the mosquito soma. PLoS Pathogens, 8(1). https://doi.org/10.1371/journal.ppat.1002470

Nasar, F., Palacios, G., Gorchakov, R. V, Guzman, H., Da Rosa, A. P. T., Savji, N., Popov, V. L., Sherman, M. B., Lipkin, W. I., Tesh, R. B., \& Weaver, S. C. (2012). Eilat virus, a unique alphavirus with host range restricted to insects by RNA replication. Proceedings of the National Academy of Sciences, 109(36), 14622-14627. https://doi.org/10.1073/pnas.1204787109

Öhlund, P., Hayer, J., Hesson, J. C., \& Blomström, A.-L. (2021). Small RNA Response to Infection of the Insect-Specific Lammi Virus and Hanko Virus in an Aedes albopictus Cell Line. Viruses, 13(11), 2181. https://doi.org/10.3390/v13112181

O'Neal, S. T., Samuel, G. H., Adelman, Z. N., \& Myles, K. M. (2014). Mosquito-borne viruses and suppressors of invertebrate antiviral RNA silencing. Viruses, 6(11), 4314-4331. https://doi.org/10.3390/v6114314

Ongus, J. R., Roode, E. C., Pleij, C. W. A., Vlak, J. M., \& van Oers, M. M. (2006a). The 50 non-translated region of Varroa destructor virus 1 (genus Iflavirus): Structure prediction and IRES activity in 
Lymantria dispar cells. Journal of General Virology, 87(11), 3397-3407.

https://doi.org/10.1099/vir.0.82122-0

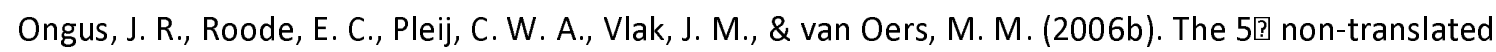
region of Varroa destructor virus 1 (genus Iflavirus): Structure prediction and IRES activity in Lymantria dispar cells. Journal of General Virology, 87(11), 3397-3407. https://doi.org/10.1099/vir.0.82122-0

Paradkar, P. N., Duchemin, J. B., Voysey, R., \& Walker, P. J. (2014). Dicer-2-Dependent Activation of Culex Vago Occurs via the TRAF-Rel2 Signaling Pathway. PLoS Neglected Tropical Diseases, 8(4). https://doi.org/10.1371/journal.pntd.0002823

Paradkar, P. N., Trinidad, L., Voysey, R., Duchemin, J. B., \& Walker, P. J. (2012). Secreted Vago restricts West Nile virus infection in Culex mosquito cells by activating the Jak-STAT pathway. Proceedings of the National Academy of Sciences of the United States of America, 109(46), 18915-18920. https://doi.org/10.1073/pnas.1205231109

Qiu, Y., Xu, Y., Wang, M., Miao, M., Zhou, H., Xu, J., Kong, J., Zheng, D., Li, R., Zhang, R., Guo, Y., Li, X., Cui, J., Qin, C., \& Zhou, X. (2020). Flavivirus induces and antagonizes antiviral RNA interference in both mammals and mosquitoes. Science Advances, 6(6). https://doi.org/10.1126/SCIADV.AAX7989

Russell, T. A., Ayaz, A., Davidson, A. D., Fernandez-Sesma, A., \& Maringer, K. (2021). Imd pathwayspecific immune assays reveal NF-kB stimulation by viral RNA PAMPs in Aedes aegypti Aag2 cells. PLOS Neglected Tropical Diseases, 15(2), e0008524. https://doi.org/10.1371/JOURNAL.PNTD.0008524

Scherer, C., Knowles, J., Sreenu, V. B., Fredericks, A. C., Fuss, J., Maringer, K., Fernandez-Sesma, A., Merits, A., Varjak, M., Kohl, A., \& Schnettler, E. (2021). An aedes aegypti-derived ago2 knockout cell line to investigate arbovirus infections. Viruses, 13(6), 1-19. https://doi.org/10.3390/v13061066

Schnettler, E., Donald, C. L., Human, S., Watson, M., Siu, R. W. C., McFarlane, M., Fazakerley, J. K., Kohl, A., \& Fragkoudis, R. (2013). Knockdown of piRNA pathway proteins results in enhanced semliki forest virus production in mosquito cells. Journal of General Virology, 94(PART7), 16801689. https://doi.org/10.1099/vir.0.053850-0

Schuster, S., Zirkel, F., Kurth, A., Cleef, K. W. R. van, Drosten, C., Rij, R. P. van, \& Junglen, S. (2014). A Unique Nodavirus with Novel Features: Mosinovirus Expresses Two Subgenomic RNAs, a Capsid Gene of Unknown Origin, and a Suppressor of the Antiviral RNA Interference Pathway. Journal of Virology, 88(22), 13447. https://doi.org/10.1128/JVI.02144-14

Siu, R. W. C., Fragkoudis, R., Simmonds, P., Donald, C. L., Chase-Topping, M. E., Barry, G., AttarzadehYazdi, G., Rodriguez-Andres, J., Nash, A. A., Merits, A., Fazakerley, J. K., \& Kohl, A. (2011a). Antiviral RNA Interference Responses Induced by Semliki Forest Virus Infection of Mosquito Cells: Characterization, Origin, and Frequency-Dependent Functions of Virus-Derived Small Interfering RNAs. Journal of Virology, 85(6), 2907-2917. https://doi.org/10.1128/jvi.02052-10

Siu, R. W. C., Fragkoudis, R., Simmonds, P., Donald, C. L., Chase-Topping, M. E., Barry, G., AttarzadehYazdi, G., Rodriguez-Andres, J., Nash, A. A., Merits, A., Fazakerley, J. K., \& Kohl, A. (2011b). Antiviral RNA Interference Responses Induced by Semliki Forest Virus Infection of Mosquito Cells: Characterization, Origin, and Frequency-Dependent Functions of Virus-Derived Small Interfering RNAs. Journal of Virology, 85(6), 2907-2917. https://doi.org/10.1128/jvi.02052-10 
Sucupira, P. H. F., Ferreira, Á. G. A., Leite, T. H. J. F., de Mendonça, S. F., Ferreira, F. V., Rezende, F. O., Marques, J. T., \& Moreira, L. A. (2020). The rnai pathway is important to control mayaro virus infection in aedes aegypti but not for wolbachia-mediated protection. Viruses, 12(8), 1-14. https://doi.org/10.3390/v12080871

Tassetto, M., Kunitomi, M., Whitfield, Z. J., Dolan, P. T., Sánchez-Vargas, I., Garcia-Knight, M., Ribiero, I., Chen, T., Olson, K. E., \& Andino, R. (2019). Control of RNA viruses in mosquito cells through the acquisition of vDNA and endogenous viral elements. ELife, 8, 1-29. https://doi.org/10.7554/eLife.41244

Torii, S., Orba, Y., Hang'ombe, B. M., Mweene, A. S., Wada, Y., Anindita, P. D., Phongphaew, W., Qiu, Y., Kajihara, M., Mori-Kajihara, A., Eto, Y., Harima, H., Sasaki, M., Carr, M., Hall, W. W., Eshita, Y., Abe, T., \& Sawa, H. (2018). Discovery of Mwinilunga alphavirus: A novel alphavirus in Culex mosquitoes in Zambia. Virus Research, 250, 31-36. https://doi.org/10.1016/j.virusres.2018.04.005

van Cleef, K. W. R., van Mierlo, J. T., Miesen, P., Overheul, G. J., Fros, J. J., Schuster, S., Marklewitz, M., Pijlman, G. P., Junglen, S., \& van Rij, R. P. (2014). Mosquito and Drosophila entomobirnaviruses suppress dsRNA- and siRNA-induced RNAi. Nucleic Acids Research, 42(13), 8732-8744. https://doi.org/10.1093/nar/gku528

Varjak, M., Dietrich, I., Sreenu, V. B., Till, B. E., Merits, A., Kohl, A., \& Schnettler, E. (2018). Spindle-E acts antivirally against alphaviruses in mosquito cells. Viruses, 10(2). https://doi.org/10.3390/v10020088

Varjak, M., Donald, C. L., Mottram, T. J., Sreenu, V. B., Merits, A., Maringer, K., Schnettler, E., \& Kohl, A. (2017). Characterization of the Zika virus induced small RNA response in Aedes aegypti cells. PLoS Neglected Tropical Diseases, 11(10), 1-18. https://doi.org/10.1371/journal.pntd.0006010

Varjak, M., Leggewie, M., \& Schnettler, E. (2018). The antiviral piRNA response in mosquitoes? Journal of General Virology, 1551-1562. https://doi.org/10.1099/jgv.0.001157

Varjak, M., Maringer, K., Watson, M., Sreenu, V. B., Fredericks, A. C., Pondeville, E., Donald, C. L., Sterk, J., Kean, J., Vazeille, M., Failloux, A., Kohl, A., \& Schnettler, E. (2017a). Aedes aegypti Piwi4 Is a Noncanonical PIWI Protein Involved in Antiviral Responses. MSphere, 2(3), e00144-17. https://doi.org/10.1128/mSphere.00144-17

Varjak, M., Maringer, K., Watson, M., Sreenu, V. B., Fredericks, A. C., Pondeville, E., Donald, C. L., Sterk, J., Kean, J., Vazeille, M., Failloux, A., Kohl, A., \& Schnettler, E. (2017b). Aedes aegypti Piwi4 Is a Noncanonical PIWI Protein Involved in Antiviral Responses. MSphere, 2(3), e00144-17. https://doi.org/10.1128/mSphere.00144-17

Vodovar, N., Bronkhorst, A. W., van Cleef, K. W. R., Miesen, P., Blanc, H., van Rij, R. P., \& Saleh, M. C. (2012). Arbovirus-derived piRNAs exhibit a ping-pong signature in mosquito cells. PLOS ONE, 7(1). https://doi.org/10.1371/journal.pone.0030861

Weaver, S. C., \& Reisen, W. K. (2010). Present and future arboviral threats. Antiviral Research, 85(2), 328-345. https://doi.org/10.1016/j.antiviral.2009.10.008 